\title{
A review on plant disease diagnosis through biosensor
}

\begin{abstract}
Plant pathogen detection is recognizing microscopic organisms such as bacteria, viruses, and fungi in quick reaction settings at nurseries, natural landscapes and micropopagation stage in infected plant tissue. Early detection provides opportunity to farmer to take proper measurement and save the crops from complete failure. For plant protection or disease control, simultaneous detection of all the present phytopathogenic microbes with quick and high accuracy is of great importance in all areas related agriculture and environmental safety. In these regard, biosensors technology in plant disease detection in broad-spectrum has advantage like lessening the investigation time and sensitivity through automation and integrating multiple processes in a single piece of equipment. The use of different types of biosensor based on colorimeter, electrochemical signal, lights emissions and nanomaterials for pioneering and sensitive biosensing systems for the recognition of pathogens is also shown. The untamed potential of various biosensors with some limitations for plant disease detection has been briefly reviewed in this article.
\end{abstract}

Keywords: biosensor, plant pathogen, disease, nanotechnology, agriculture
Volume 7 Issue 2 - 202 I

\section{Prittesh Patel}

C G Bhakta Institute of Biotechnology, Uka Tarsadia University, India

Correspondence: Prittesh Patel, C G Bhakta Institute of Biotechnology, Uka Tarsadia University, Bardoli Maliba Campus, Surat, Gujarat, India, Tel +9| 99|36688|2,

Email pritteshpatel@gmail.com

Received: February 01, 202I | Published: April 26, 2021

\section{Introduction}

A biosensor is an analyte detecting machine containing a bioelement recognition component and a transducer. A bio- recognition component of biosensor particularly recognizes and communicates with an analyte, and the changes in its physicochemical properties are usually transformed into an electrical signal by a transducer. ${ }^{1}$ The bio-recognition component may contain catalyst, microorganisms, antibodies, DNA and RNA. Communicable plant diseases are caused by pathogenic microbes such as fungi, bacteria, viroids, viruses, phytoplasma and nematodes. ${ }^{2}$ Globally, plant pathogen infections are amongst chief biotic factors limiting plant yield and causes financial losses to farmers as well as plant based industries. It was accounted for that around $20-30 \%$ of the field crops are yearly lost because of infections. ${ }^{3,4}$ Traditional techniques, for example, isolation on specific media are valuable for the pathogenic microorganism's identification yet subject to restrictions because of the way that numerous pathogens are covered by abundance of more quickly growing microorganisms. Besides, these strategies introduce the downsides of dreary method, requirement for trained personnel and long time to yield accurate result. So, the conventional methods of detecting pathogens are timeconsuming and expensive for the farmers in rural areas. Detection of causal agent of the plant disease is most crucial as primary step to handle a plant disease in field. Many of the pathogenic microbes such as virus and phytoplasma are seed borne and identification such pathogen at the tissue culture stage can prevent major crop loss. In the previous decade, the interest for quick and precise on location identification of plant disease has expanded because of developing new mutant pathogens with resistance to pesticides and overuse of toxic chemicals to prevent spread of diseases. Currently major serological techniques used to identify phytopathogens consist of direct tissue blot immunoassays and ELISA. ${ }^{2}$ In this technique, the specific antigens from the infectious microbes are made to explicitly bind with antibodies conjugated to a protein. The recognition can be envisioned in view of colour changes because of the association between the substrate and the immobilized protein. The execution of ELISA can be enhanced significantly with the utilization of particular monoclonal and recombinant antibodies which are monetarily accessible.

Further DNA-based techniques such as polymerase chain reaction and dot blot hybridization have also been used for pathogen identification and detection. PCR based detection requires sample preparation, DNA extraction, portable and accurate temperature control system, and sample evaporation concerns in open devices. ELISA and PCR both these procedures are tedious and require complex instruments, being not reasonable for in-situ investigation. In the previous decade, the interest for fast and exact on-site detection of plant disease determination has expanded because of arising microbes resistance to pesticides. So, there is solid enthusiasm for growing new bio-sensing systems for early recognition of plant infections with high affectability and specificity at the purpose of-mind. In this unique situation, here the current headway of favourable bio-sensing systems for plant pathogen recognition is discussed.

\section{Biosensor based diagnosis}

When plants are exposed to pathogens they initiate protection reactions whose molecular mechanisms are very complex. At the early stages, when visual symptoms such as injuries on the leaf surface are absent, plants respond to the presence of a pathogen with physiological component such as the decrease of the photosynthesis rate, which induces an increase of fluorescence and heat emission. ${ }^{4}$ The possibility to distinguish distinctive contaminations in a similar plant is attractive, since plants can be influenced at the same time by numerous pathogens, for example, nematodes, fungi, bacteria, phytoplasmas, viruses and viroids that conventional strategies identify at a late symptomatic stage. Globally plant pathogenic microbes cause various infectious diseases that are flattering more and more serious. Till date many researchers have tried to developed different types of biosensors with range of approaches. Detection of plant infection utilizing electrochemical techniques has pulled in much intrigue in light of their basic instrumentation, high specificity, affectability, quick, and is economical with potential for applications in sub-atomic sensing instrument. ${ }^{5,6}$ Recently, nanomaterial-based electrochemical 
sensors have been reported for plant disease detection by Umasankar et al. ${ }^{7}$ The utilization of gold nanoparticle (AuNP) modified cathode for the electrochemical detection of methyl salicylate, a key plant volatile organic compound released by plants during infections. In case of DNA based electrochemical strategies, voltammetric examinations have been utilized as a basic device for a discriminative investigation of nucleic acid conformation and modification with the synchronous identification of all bases of the DNA without the need of a hydrolysis step. ${ }^{8,9}$ The detection generally includes the redox labels physically or covalently linked to specific target DNA allocated on the active electrode surface. ${ }^{10}$ Current advance in DNA based biosensors for improvement of parallel microarrays and high-throughput outlines can be connected to DNA sequencing innovations. Although much research has been done on electrochemical biosensors for other areas like food quality but its practical application for plant disease detection is in pipeline for detailed investigation.

Colorimetric biosensors are an appealing optical biosensor since one can undoubtedly and immediately see with the bare eye the presence of pathogenic microorganisms in the specimen through a colour change without the requirement for any expository instrument or chemical reagent. Optical biosensors measure light assimilated or radiated as the after effect of a biological or synthetic response, while electrochemical biosensors depend on biochemical responses that cause electron exchange between a functionalized electrode and an analyte in solution, and can make amperometric, voltammetric, or impedimetric estimations. ${ }^{11}$ Another approach, nanotechnology has real way against numerous agricultural problems including plant disease identification as well as control. Nanoparticles show interesting electronic and optical properties and can be incorporated utilizing various kinds of materials for electronics and detecting applications. ${ }^{12}$ The prevalence of nanomaterials for sensor improvement could be credited to the friendly platform it facilitate the gathering of bio-recognition component, the high surface region, high electronic conductivity and plasmonic properties of nanomaterials that upgrade the constraint of detection..$^{13}$ Now a day's nano-based materials is introduced which enhance the effectiveness of fungicides and pesticides, enabling minor dosages to be utilized..$^{14}$ Additionally, nanodiagnostic and microfluidics offer novel tools to improve the sample preparation step that remains difficult to incorporate in a miniaturized platform. The signal intensification methodologies may perhaps challenge those of target enhancement. Fast nearby recognition of plant pathogens utilizing nanosensor, nanobased kits, nanobarcodes, nanobiosensors and other portable diagnostic systems can help agricultural and food industry to manage different plant diseases. The Cucumber Mosaic Virus (CMV) and Papaya Ring Spot Virus (PRSV) is a highly aggressive disease that can reduce yield and quality of the vegetable and fruit. Ariffin et al introduced fabrication of nanowire transducer using photolithography and its function in detection of CMV and PRSV viruses. ${ }^{14}$ Enzyme-based biosensors are based on using enzymes that are specific to the biomolecules under recognition to catalyze the generation of a product that can be evaluated by a transducer. ${ }^{15,16}$ A large portion of the enzyme utilized in biosensors is oxidases that respond with dissolved oxygen to produce hydrogen peroxide.

Antibody-based biosensors with a range of transducing methods have been reported by researchers. ${ }^{17,18}$ Detection of phytopathogenic organism by immunological techniques depends on availability and affinity of selective antibody binding to the target bio-molecule. In case of complex plant material, sample is prepared by separation and accumulation of target molecules to facilitate effective pathogen sensing. ${ }^{19}$ Antibody-based sensors, also known as immunosensors involve the use of both polyclonal and monoclonal antibodies. Antibodies can be directly immobilized on the exterior of the transducer or attached to the surface of magnetic beads to perform immunomagnetic separation and detection. Conversely limitations of a lot of antibodies based biosensors comprise specific binding with a particular antigen, issues such as the exposure of a bacterial strain to environmental stress such as $\mathrm{pH}$, could cause errors in the measurement. Utilizing biosensors and other compact demonstrative frameworks can definitely help the agriculture business. However several drawbacks of biosensor technology for on-site diagnosis of multiple pathogens includes sample preparation, limited life span of biological entity, weak selectivity in complex sample matrices, complexity of manipulations and obviously the high cost.

\section{Future work}

In spite of the fact that, plant biosensors based research has acquired importance without a doubt, recently, there is less research available. Also, likely potential demonstration of Nano-Inspired Biosensors for non-plant applications don't limit plant biosensors to move foot in the presence, of contemporary procedures or 'Transcriptomic Biosensors', 'Hereditarily Encoded Biosensors' and Chimeric Biosensing technologies. ${ }^{20}$ The addition of more novel sensors for disease recognition, for example, optical fiber biosensors and electrochemical biosensors will carry a more advantageous way to deal with the detecting of plant infections. Considering the significance of monitoring plant health and status of present nanobiosensors, on a flipside drawback of presently available techniques, advance nano materials and novel biomarkers, an appropriate kick start is expected to urge scientist to redirect their consideration for monitoring plant health and growth.

\section{Conclusion}

One of the generally confronted difficulties across the globe incorporates loss of the significant part of crop yield because of diseases. After providing effective resources to the fields, significant part of the yield is reduced by the presence of pathogens in the plants. This prompts center around successful methods of identification of disease in plants. To give powerful measures to detection and avoidance of the destruction requires an early identification of type of plant disease present. Conventional methods such as microscopic examination of diseased tissue or culturing to detect phytopathogens are time consuming and required skilled person while new technique like biosensor allows easy and fast detection. The interest for biosensors for identification of plant pathogenic microbes has expanded and investigation is centred on development of small portable devices that would permit quick, precise, and on location recognition. Biosensor technology in combination with synthetic biology is a promising topic of research for agriculture scientist.

\section{Acknowledgements}

None.

\section{Conflicts of interest}

The authors declare that there is no conflict of interest.

\section{References}

1. Srinivasan B, Tung S. Development and applications of portable biosensors. Journal of laboratory automation. 2015;20(4):365-389. 
2. Khater M, De la Escosura-Muñiz A, Merkoçi A. Biosensors for plant pathogen detection. Biosensors and Bioelectronics. 2017;93:72-86.

3. Oerke E. Crop losses to pests. The Journal of Agricultural Science. 2006; 144:31.

4. Sankaran S, Mishra A, Ehsani R, et al. A review of advanced techniques for detecting plant diseases. Computers and Electronics in Agriculture. 2010;72(1):1-13.

5. Wei F, Lillehoj PB, Ho CM. DNA diagnostics: nanotechnologyenhanced electrochemical detection of nucleic acids. Pediatric research. 2010;67(5):458-468.

6. Yin BC, Guan YM, Ye BC. An ultrasensitive electrochemical DNA sensor based on the ssDNA-assisted cascade of hybridization reaction. Chemical Communications. 2012;48(35):4208-4210.

7. Umasankar Y, Ramasamy RP. Highly sensitive electrochemical detection of methyl salicylate using electroactive gold nanoparticles. Analyst. 2013;138(21):6623-6631.

8. Oliveira-Brett A, Piedade J, Silva Ld, et al. Voltammetric determination of all DNA nucleotides. Analytical biochemistry. 2004;332(2):321-329.

9. Pedano M, Rivas G. Immobilization of DNA at glassy ccarbon electrodes: A critical study of adsorbed layer. Sensors. 2005;5(6):424-447.

10. Wongkaew P, Poosittisak S. Diagnosis of sugarcane white leaf disease using the highly sensitive DNA based voltammetric electrochemical determination. American Journal of Plant Sciences. 2014.

11. Martinelli F, Scalenghe R, Davino S, et al. Advanced methods of plant disease detection. A review. Agronomy for Sustainable Development. 2015;35(1):1-25.
12. Shipway AN, Katz E, Willner I. Nanoparticle arrays on surfaces for electronic, optical, and sensor applications. Chem Phys Chem. 2000;1(1):18-52.

13. Fang Y, Ramasamy RP. Current and prospective methods for plant disease detection. Biosensors. 2015;5(3):537-561.

14. Ariffin SA, Adam T, Hashim U, et al. Plant diseases detection using nanowire as biosensor transducer. Advanced Materials Research. 2014

15. Katrlík J, Pizzariello A, Mastihuba Vr, et al. Biosensors for L-malate and L-lactate based on solid binding matrix. Analytica chimica acta 1999;379(1-2):193-200.

16. Vidal JC, Espuelas J, Garcia-Ruiz E, et al. Amperometric cholesterol biosensors based on the electropolymerization of pyrrole and the electrocatalytic effect of Prussian-Blue layers helped with selfassembled monolayers. Talanta. 2004;64(3):655-664.

17. Abdul Kadir MK, Tothill IE. Development of an electrochemical immunosensor for fumonisins detection in foods. Toxins. 2010;2(4):382 398.

18. Jacobs M, Panneer Selvam A, Craven JE, et al. Antibody-conjugated gold nanoparticle-based immunosensor for ultra-sensitive detection of troponin-T. Journal of laboratory automation. 2014;19(6):546-554.

19. Gehring AG, Tu SI. High-throughput biosensors for multiplexed foodborne pathogen detection. Annual review of analytical chemistry. 2011;4:151-172.

20. Kumar V, Arora K. Trends in nano-inspired biosensors for plants. Materials Science for Energy Technologies. 2020;3:255-273. 